\title{
The Spreading of Droplets on Solids
}

\author{
Kimmo Kaski \\ Tampere University of Technology, Tampere, Finland
}

High-precision experiments reveal unusual molecular-level features of precursor films that precede the interfaces of liquid droplets advancing across atomically flat and inert surfaces. Understanding has improved because microscopic models are now able to reproduce the features.

The spreading of liquid droplets on surfaces plays a critical role in numerous technologies including lubrication, painting, emulsion, dying, and oil recovery from porous rocks. As long ago as 1919, Hardy suggested that the spreading of a volatile drop of liquid on a surface manifested a remarkable phenomenon whereby the macroscopic motion of the drop is preceded by a film, called a precursor, of molecular thickness. This model was confirmed in 1989 by Heslot et al. [1] in outstanding studies of the spreading of small but practically nonvolatile droplets of silanols (silicon oils) across atomically flat and inert silicon wafers. The experiments also revealed for the first time the striking phenomenon of dynamic layering, which appears to have little if anything to do with equilibrium layering seen at surfaces in situations of partial wetting. Moreover, there was evidence for the formation of a surface gas on the substrate during the final stages of spreading.

These experimental observations have not been corroborated fully by theoretical considerations of the hydrodynamics [2] so the dynamics of droplet spreading remains poorly understood at the microscopic and molecular levels. The phenomenon has therefore experienced a tremendous surge in interest these last few years. This short presentation aims to show that recent experimental results are starting to be better understood using microscopic models based on computer simulation.

\section{Experimental Observations}

The recent experiments of Heslot et al. [1] used sophisticated techniques such as ellipsometry to measure profiles of spreading droplets as a function of time. The methods

Kimmo Kaski has been Professor of Computational Science and Engineering at Tampere University of Technology, POB 692, FIN-33101 Tampere, Finland, since 1987. He graduated in electrical engineering at the Helsinki University of Technology and received a D.Phil. in theoretical physics from Oxford University in 1981 before working as a postdoc at Temple University (Philadelphia, USA), Geneva University and the Kernforschungszentrum Jülich. Professor Kaski has held visiting positions at Temple University, Oxford University, Southern Illinois University, University of Mexico, and University of Helsinki. yield a very high vertical resolution (down to sub-atomic level) while the horizontal resolution is in the millimetre range. Experiments were carried out by placing sub-nanolitre volume droplets of very viscous polydimethyliloxane (PDMS) or tetrakis(2-ethylexoxy)silane (TEHOS) onto atomically flat and inert surfaces (silicon wafers). Very high viscosity sets the timescale of spreading to be tens or even hundreds of hours, thus highlighting the degree of sophistication needed to control the ambient conditions during measurements.

\section{Morphological features}

The formation of a precursor film formation with a thickness of about $5 \AA$ and a width of more than $5 \mathrm{~mm}$ is clearly seen in Fig. 1a for the case of PDMS. Moreover, the long tail of the precursor front represents evidence that the final state of spreading is not a pancake, as originally proposed by Hardy, but rather a surface gas owing to the domination of molecular diffusion over liquid cohesion.

Dynamic structuring of the fluid as series of distinct molecular layers was observed for TEHOS (Fig. 1b). The layers, extending up to four molecular distances from the substrate surface, are caused by silicon acting as a high-energy surface for TEHOS to give a long-range force between the substrate and the molecules in the drop. The gravitational force is negligible for the droplet dimensions in question, so this attractive force drawing the molecules towards the surface competes with the cohesive force that tries to hold the droplet together. It is in fact the strength of the surface interaction, which is proportional to the so-called Hamaker constant, that distinguishes cohesion-dominated partial wetting from adhesion-dominated complete wetting and spreading.

\section{Spreading mechanisms}

Explaining the kinetics of spreading at early and late times, in other words, the nature of the dominant mechanisms of spreading, is a crucial issue. The time dependence of the width $W$ of the precursor film is of particular interest, and most experiments have established that the diffusive law $W \propto t^{1 / 2}$ arises at late times, with the exception of squalane [1] which revealed significant departures from this law at early times.

Hardy's explanation for precursor spreading was based on a precursor comprising vapour evaporated from the droplet and condensed on the substrate. Reminiscent of this model is Forcade and Mate's [3] rationale for ellipsometry observations of dynamic layering in TEHOS in terms of evaporation and condensation processes. In addition, these authors found that the layering manifested itself as a layer-by-layer evaporation (a layer-by-layer peeling-off) of an initially wedge-shaped fluid sample.

In contrast to the idea of volatility playing the key role in droplet spreading, Joanny and de Gennes [4] described the process in terms of the microscopic hydrodynamics of non-volatile fluids. The basic assumption here is that fluid films are at least a few tens of Annströms thick. According to Joanny
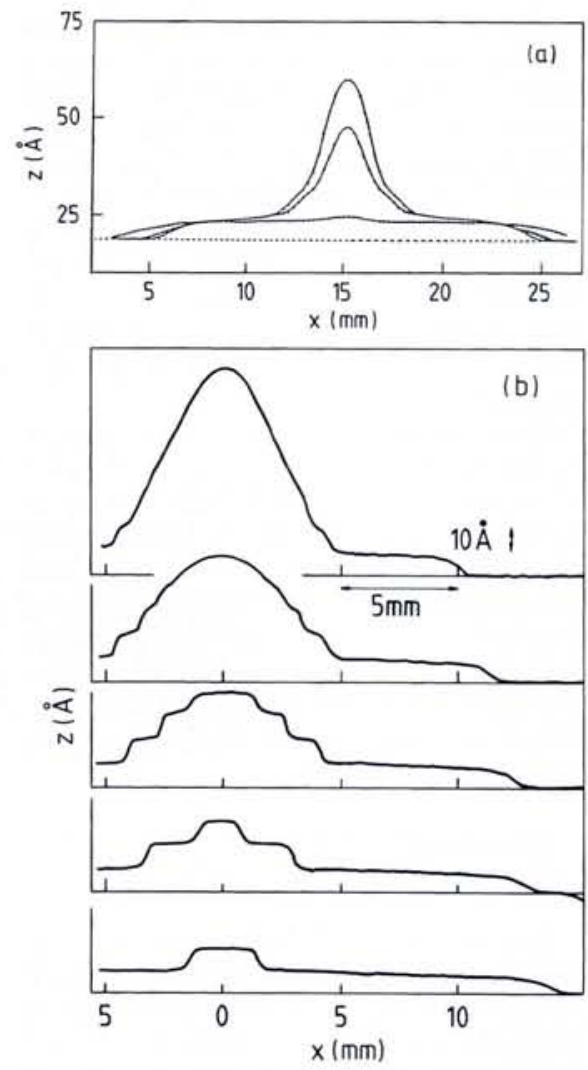

Fig. 1 - The thickness profiles of droplets of silicon oils on silicon wafers at ambient temperature as measured using ellipsometry by Heslot et al. [1]. a, upper) PDMS droplet with a volume of 0.19 nanolitre at 47 hours (upper curve), 56 hours (middle curve) and 96 hours (lower curve). The dashed line indicates the position of the $\mathrm{SiO}_{2}$ surface. $b$, lower) TEHOS droplet with a volume of 0.47 nanolitre at 71,83 , 118,144 , and 168 hours, respectively from top to bottom. The baseline corresponds to the position of the $\mathrm{SiO}_{2}$ surface. 

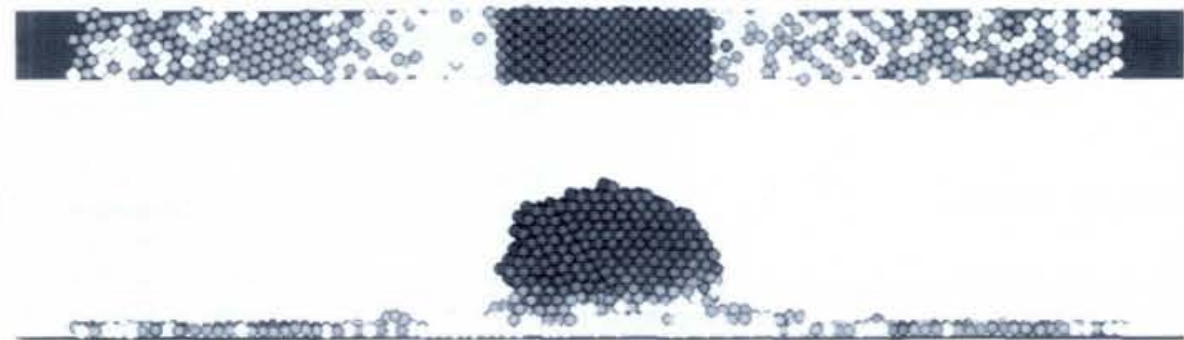

Fig. 2 - Snapshots from above (upper) and as a vertical cross-section (lower) of a molecular dynamics simulation of a fluid of Lennard-Jones particles introduced as a cylindrical droplet ridge on top of a flat, unstructured substrate with a van der Waals-type long-range potential. Three levels of grey scale are used to indicate the velocity of the particles (dark particles are moving at the lowest velocity and white particles at the highest velocity in a predominantly horizontal direction).

and de Gennes, the development of the precursor results, on one hand, from the drift of the macroscopic edge of the droplet profile (also called the foot) and, on the other hand, from expansion of the well-developed precursor film which is being driven by unbalanced capillary forces. Their analysis shows that the drift-dominated early time spreading shows a growth law with a dependence with time which is a little less than linear $(W \propto$ $t^{9 / 10}$ ). At late times, it changes to diffusive behaviour which is driven by expansion of the film [5]. In the cross-over regime, drift and diffusion occur simultaneously. However, hydrodynamics has not been able to account for the phenomenon of dynamic layering.

So although the theory of the macroscopic motion of a fluid is well established $[2,4]$, the same cannot be said for the precursor film. Indeed, it has become evident from the recent experimental results that our understanding of microscopic spreading mechanisms and their characteristic behaviour with time remains poor. Moreover, interpretations of various experiments seem contradictory. Microscopic or molecular models are therefore desirable.

\section{Microscopic Models}

Solid-on-solid strings

The first attempt to improve understanding using a microscopic approach was made by Abraham et al. [6] who developed an interfacial model for a strictly non-volatile fluid wedge. The interface was analysed in terms of Langevin dynamics for the displacement of horizontal solid-on-solid (HSOS) strings at increasing heights from the substrate. A free-energy function associated with any configuration of the interface forming strings revealed a competition between surface tension and substrate interaction, where the latter could extend over a long range. In the Langevin dynamics, the Brownian fluctuation of an interface element was introduced as white noise. The model was sufficiently simple that it could be studied analytically. In the case of complete wetting owing to an adequately strong but short range (up to first layer) surface interaction, a precursor monolayer was shown to extract itself from the interface and advance at a constant velocity. The remainder of the interface advanced with a $W \propto(t \log t)^{1 / 2}$ growth law. Both of these growth laws were subsequently verified by numerically integrating the Langevin equation of motion [7].

When the substrate interaction was extended up to the second layer, two cases could be identified depending on the relative strengths of the interactions. The first two layers could move together at a constant velocity thus forming a thicker precursor film. Alternatively, the precursor film could consist of the first layer moving at a constant velocity followed by a second layer moving at a constant but smaller velocity. This idea could be extended for even longer range surface potentials with similar results. There is not yet any experimental evidence for the first type of spreading mechanism. However, the latter mechanism models the dynamic layering that was observed using ellipsometry.

Next came a numerical study made by changing the Langevin dynamics to a dynamics based on a master eqution representing a probabalistic description of the lattice gas. The HSOS Hamiltonian was similar to that used in the Langevin theory, but the substrate potential was chosen to be either short range or of the long-range van der Waals type given by $V(z)=-A / z^{3}$, where $z$ is the distance from the substrate and $A$ is the strength of the potential corresponding to the Hamaker constant. The simulations were done with the so-called importance sampling Monte Carlo method where the most proable samples of the system configuration are taken, and in which the system is driven towards equilibrium using the detailed balance condition. In the case of a short-range potential, all the results [8] confirm very precisely the predictions of the analysis based on the Langevin theory. The same is also true for the van der Waals-type substrate potential in that dynamic layering and a thick precursor film were predicted.

\section{Molecular dynamics}

In these two models based on a HSOS Hamiltonian the fluid is strictly non-volatile. In addition, discretization was assumed, at least vertically for the Langevin theory and in all directions for the Monte Carlo model. A natural next step in modelling therefore involves relaxing both of these limiting assumptions. This can be done with molecular dynamics in which particles are assumed to interact through the Lennard-Jones pairpotential and to move about with continuum (Newtonian or non-Newtonian) dynamics. In a study by Yang et al. [9], the fluid and the solid substrate were both composed of particles of similar size, but with the substrate particles having a much larger interaction strength. This entailed a strongly varying potential between the fluid and substrate particles, and a highly volatile fluid making the substrate moistened by fluid particles. However, these conditions do not correspond to those used in the experiments by Heslot et al., which may explain the prediction that the precursor spread very slowly with $W \propto(\log t)^{1 / 2}$.

Nieminen et al. [10] also used molecular dynamics with the same Lennard-Jones pair-potential between fluid particles, but assumed the substrate to be a flat, unstructured continuum so as to model the very smooth $\mathrm{SiO}_{2} /$ silicon wafers used in the experiments. The fluid molecules - which in reality are considerably larger than the atoms of the substrate - thus have an interaction with the substrate of the form $V(z)=$ $-A / z^{3}+B / z^{9}$, where $A$ plays the role of the Hamaker constant and $B$ is also a constant. The dynamics were assumed to be nonNewtonian by incorporating a Nose-Hoover heat sink to model the friction (the rapid dissipation of energy) experienced by the fluid particles. The parameters for the molecular dynamics simulations were chosen to make the droplet practically non-volatile, i.e., the wetting proceeded on a dry substrate. This can be seen in Fig. 2 giving a snapshot of the distribution of particles during a simulation experiment, where the particles are found to be either in the droplet cap or in the spreading precursor film. The figure also reveals the presence of a second fluid layer on top of the precursor layer. This serves as evidence for dynamic layering in a continuum model - layering which was to a certain sense built into the lattice models.

The growth kinetics of the precursor layer were found to be most interesting, since for early times the growth rate seemed to be almost constant before crossing over to diffusive spreading at late times. As mentioned above, the diffusive behaviour corresponding to the macroscopic drop feeding the precursor has been seen in the ellipsometric experiments. But this is not (yet) the case for the approximately linear growth law, where spreading is driven by the flow of material from the cap to the film. 


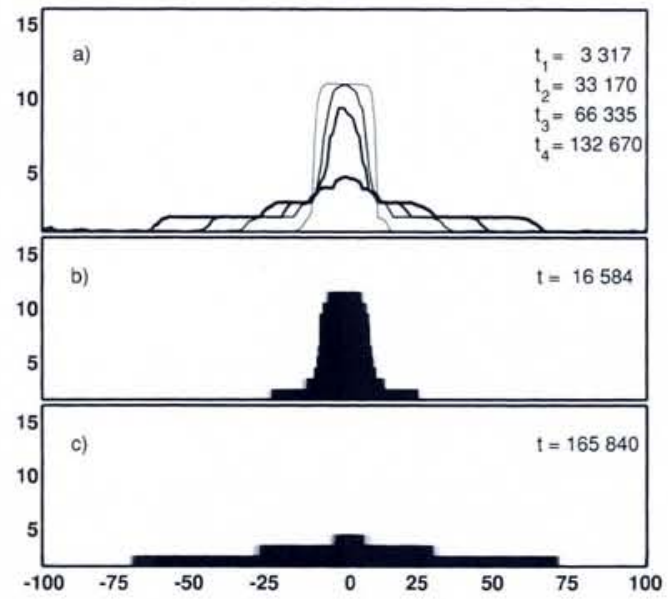

Lattice-gas model

The molecular dynamics analysis remains computationally very heavy despite ignoring the substrate structure. Moreover, the recent ideas about the spreading mechanisms put forward by Forcade and Mate need further investigation and clarification. For these reasons we returned to the Monte Carlo method but used an Ising or lattice-gas model that is richer from a statistical mechanics point of view [11]. The fluid particles were assumed to interact with their nearest neighbours in a cubic lattice and with the flat, unstructured substrate through the long-range potential $V(z)=-A / z^{3}$. The dynamics were assumed to obey Kawasaki spinexchange dynamics, which conserves the amount of fluid matter in the simulation system. The model therefore allows for volatility, the amount of which can be changed by adjusting the Hamaker constant $A$ and the temperature in the simulations.

As in the molecular dynamics simulations, we found dynamic layering and linear precursor growth at early times crossing over to diffusive spreading at late times. These features are shown in Fig. 3 giving snapshots of the development with time of the profile of an initially ridge-shaped droplet profile as well as the average densities of the droplet for early and late times.

\section{Tracer Studies}

We have studied the fluxes at different times of tracer particles within the fluid phase and through the vapour phase via the evaporation-condensation mechanism. At early times, when growth is linear or nearly so, the flow of matter is concentrated in the fluid interface region of the droplet cap and the flux of particles is predominantly vertically downwards towards the substrate. This results in fast precursor growth and a purely horizontal flux in the precursor. For late times, when growth is diffusive, the fluxes are purely horizontal in the precursor and predominantly horizontal in the second and third layer. Increased vertical fluxes were only observed in the neighbourhood of the layer edges. Under these conditions, growth of the precursor is dominated by particles migrating off from the second layer edge and performing random walks on the precursor layer until they find holes to fill or rough precursor edges to stick with. It is believed that these mechanisms explain the experi-

Fig. 3 - Snapshots from a Monte Carlo simulation of the development with time of a rectangular ridged-shaped initial droplet made from a three-dimensional lattice gas [11]. The droplet (thickness) profile (a, upper) as given by the droplet interface corresponding to half the maximum fluid density. Average densities of the droplet are plotted for early ( $b$, middle) and late (c, lower) times.

mental results of Forcada and Mate more satisfactorily than the evaporation-condensation process these authors invoked.

The models that have been discussed assume fluid molecules without any geometric structure. However in some silicon oils the molecules have a clear chain-like structure which implies that excluded volume and chain entanglement need to be taken into account. These aspects, which are treated fully in [12], essentially involve details beyond the scope of this brief review of the status of microscopic treatments of droplet spreading

[1] Heslot F., Fraysse N. \& Cazabat A.M. Nature (London) 338 (1989) 640; Heslot F, Cazabat A.M. \& Levison P., Phys. Rev. Lett. 62 (1989) 1286

[2] de Gennes P.G., Rev. Mod. Phys. 57 (1985)
827.

[3] Forcada M.L. \& Mate M., Nature (London) 363 (1993) 527.

[4] Joanny J.F. \& de Gennes P.G., J. Phys. (Paris) 47 (1986) 121

[5] Cazabat A.M., Contemp. Phys. 28 (1987) 347.

[6] Abraham D.B., Collet P., de Coninck J. \& Dunlop F., Phys. Rev. Lett. 65, 195 (1990); J. Stat. Phys. 61 (1990) 509.

[7] Chopard B., J. Phys. A 24 (1991) L345

[8] Abraham D.B., Heinio J. \& Kaski K., J. Phys. A 24 (1991) L309; Phys. Rev. B 45 (1992) 4409

[9] Yang J.X., Koplik J. \& Banavar J.R., Phys. Rev. Lett. 67 (1991) 3539; Phys. Rev. A 46 (1992) 7738

[10] Nieminen J., Abraham D.B., Karttunen M. \& Kaski K., Phys. Rev. Lett. 69 (1992) 124.

[11] Lukkarinen A., Kaski K. \& Abraham D.B., Phys. Rev. E. (1995).

[12] Albrecht U., Otto A. \& Leiderer P., Phys. Rev. Lett. 68 (1992) 3192; Nieminen J.A. \& Ala-Nissila T., Europhys. Lett. 25 (1994) 593; idem, Phys. Rev. E 49 (1994) 4228.

\title{
A TRANSFERABLE TIGHT-BINDING POTENTIAL FOR TRANSITION METAL SILICIDES
}

\section{The Intriguing Case of $\mathrm{FeSi}_{2}$}

\author{
Leo Miglio \\ Physics Department, University of Milan
}

Semi-empirical methods for electronic structure calculations which combine flexibility and limited computational requirements allow for a reliable description of the link between structural features and the physical properties of technologically relevant complex materials.

A renaissance of the well-established tightbinding method for the semi-empirical calculation of electronic states in solids has taken place in the last few years owing to its ability to provide reliable interatomic potentials for total energy and molecular dynamics calculations in semiconductors and carbon structures [1]. The method has now been extended to cover transition-metal silicides, an important class of materials, both scientifically and technologically.

Metallic silicides play an increasingly important role in the fabrication of microelectronic devices owing to their excellent compatibility with technologies for manufacturing integrated circuits based on silicon Refractory disilicides such as $\mathrm{WSi}_{2}$ and

Leo Miglio has been an Associate Professor in the Physics Department, University of Milan, via Celoria, 16, I-20133 Milan, since 1993. He studied at the University of Pavia and after graduating in 1980 from the ICTP, Trieste, took up a postdoc position in the University of Milan before becoming an Assistant Professor in 1983. He has held visiting positions at the Max-Planck Institut für Strömungsforschung (Göttingen), the Instituto de Ciencias de $\mathrm{Ma}$ teriales (Madrid) and Florida State University (Tallahassee, USA).
$\mathrm{TiSi}_{2}$ are used at present, but their poor crystallographic matching with silicon generates defect-rich interfaces. On the other hand, near-noble disilicides such as $\mathrm{NiSi}_{2}$ and $\mathrm{CoSi}_{2}$ with the simple fluorite $\left(\mathrm{CaF}_{2}\right)$ structure display very sharp interfaces with silicon, to the extent that epitaxial $\mathrm{CoSi}_{2}$ has been experimented with for transistors of the permeable-basis type [2]. Attention of a different sort has recently been devoted to another member of the same family of compounds - namely $\mathrm{FeSi}_{2}$ - because it exists as a semiconducting ( $\beta$-) phase at room temperature. This phase has a distorted fluorite structure with a large number (48) of atoms in the (orthorhombic) unit cell. Being very complex, it can only be dealt with using semi-empirical methods and thus represents a meaningful example of the predictive power of tight-binding potentials.

The possibility of using $\beta$ - $\mathrm{FeSi}_{2}$ for optoelectronic devices has been explored. However, this application is partially frustrated by light absorption measurements and firstprinciples calculations [3] showing that an indirect band gap as large as $0.78 \mathrm{eV}$ is present, a value which is slightly smaller than the direct gap at the Brillouin zone centre $(0.85 \mathrm{eV})$. Secondly, owing to the difference 